\title{
Incisuroplastia troclear e estabilização articular após transeç̧ão do ligamento cruzado cranial. Estudo clínico e radiográfico em cães
}

\author{
Intercondylar notchplasty and fascial strip repair following transection of the cranial cruciate ligament. \\ Clinical and radiographic aspects in the dog
}

\author{
André Luis Selmi ${ }^{I}$ João Guilherme Padilha Filho ${ }^{\text {II }}$ Bruno Testoni Lins ${ }^{\text {III }}$ \\ Guilherme Maia Mendes ${ }^{\text {III }}$ Gisleine Cristina Eimantas ${ }^{\mathrm{I}}$
}

\section{RESUMO}

Avaliaram-se as alterações clínicas e radiográficas em nove cães adultos, após a transecção do ligamento cruzado cranial (LCCr) seguida da substituição ligamentar por retalho de fáscia lata, associada ou não à incisuroplastia troclear (ITR). O joelho direito (GI) foi submetido à ITR e posterior estabilização articular, e o esquerdo somente à substituição ligamentar (GC). Os animais foram avaliados nos períodos pré-operatório, pós-operatório (po) imediato e aos 30, 90 e 180 dias de po, período coincidente com o momento de eutanásia de subgrupos de três cães. A instabilidade articular e o grau de claudicação diminuíram significativamente durante o período de avaliação, apesar da instabilidade persistir durante a flexão articular. Observou-se decréscimo significativo do perímetro muscular da coxa aos 30 e 90 dias p.o. em ambos os grupos. Não foram evidenciadas alterações nos graus de extensão e flexão articulares, na rotação interna da tíbia, na amplitude de movimento articular ou de doença articular degenerativa (DAD) durante o período de avaliação em ambos os grupos. Conclui-se que a ITR, associada à técnica de estabilização articular, não produz alterações em nenhuma das variáveis estudadas em cães, quando comparada à estabilização intra-articular, isoladamente.

Palavras-chave: incisura troclear, doença articular degenerativa, ligamento cruzado cranial, canino.

\section{ABSTRACT}

Clinical and radiographic changes associated with intercondylar notchplasty (IN) after transection of the cranial cruciate ligament (CCL) followed by a fascial strip reconstruction were studied in nine adult dogs. The right stifle was submitted to IN followed by fascial strip reconstruction of the CCL (GI) while in the left IN was not performed (GC). Dogs were evaluated the day prior to surgery, on the immediate postoperative day and at 30, 90 and 180 days after surgery, time at which a subgroup of three dogs were euthanatized. Instability and lameness significantly decreased throughout the evaluation period, although instability was observed during flexion. There was a significant decrease in muscle perimeter at 30 and 90 days po in both groups. Changes in degree of extension, flexion, internal rotation of the tibia, range of motion, or DAD were not observed throughout the evaluation period in any group. It is concluded that IN, followed by a fascial strip reconstruction of the CCL does not cause any change in the variables studied in dogs, when compared to a fascial strip reconstruction alone.

Key words: intercondylar notch, degenerative joint disease, cranial cruciate ligament, canine.

\section{INTRODUÇÃO}

A ruptura do ligamento cruzado cranial (RLCCr) é a causa mais freqüente de claudicação em cães (AIKEN et al., 1992; INNES \& BARR, 1998). Decorre de alterações degenerativas do próprio ligamento (ARNOCSKY, 1996; MOORE \& READ, 1996), embora modificações da conformação óssea (READ \& ROBINS, 1982) e trauma, como a hiperextensão e rotação

ICurso de Medicina Veterinária, Universidade Anhembi Morumbi. Endereço para correspondência: Rua Conselheiro Lafaiete, 64, Brás, 03164-110, São Paulo, SP, Brasil. E-mail:selmi@anhembi.br.

"Departamento de Clínica e Cirurgia Veterinária, Faculdade de Ciências Agrárias e Veterinárias (FCAV), Universidade Estadual Paulista (UNESP), Jaboticabal, SP, Brasil.

"I'Curso de Pós-graduação em Cirurgia Veterinária, Faculdade de Medicina Veterinária e Zootecnia (FMVZ), UNESP, Botucatu, SP, Brasil. 
interna da tíbia, também sejam implicados (MONTGOMERY et al., 1995).

Clinicamente, após a RLCCr, observa-se claudicação em graus variados, acompanhada por dor (MOORE \& READ, 1996). O exame físico revela diferentes graus de atrofia muscular da coxa, crepitação articular, redução da amplitude de movimento articular em decorrência da DAD e espessamento da cápsula articular (VASSEUR \& BERRY, 1992). O diagnóstico clínico é baseado na presença do movimento de gaveta ou pelo deslocamento cranial da tíbia durante o teste de compressão tibial (MOORE \& READ, 1996). Entretanto, o diagnósticodefinitivo é realizado durante o ato operatório, quando se observa a falta da integridade da estrutura (SHIRES \& HULSE, 1984; COETZEE \& LUBBE, 1995). Radiograficamente observam-se efusão articular e diminuição da área do coxim infrapatelar nos casos agudos e espessamento da cápsula articular medial, presença de osteófitos e esclerose óssea subcondral em animais após 21 dias da lesão (ELKINS et al., 1991).

Vários estudos, experimentais ou clínicos, têm buscado avaliar os resultados das técnicas de substituição ligamentar por enxertos autógenos (SHIRES \& HULSE, 1984; ELKINS et al., 1991; VASSEUR \& BERRY, 1992; COETZEE \& LUBBE, 1995; GEELS et al., 2000; MELO, 2001), alógenos, ou substitutos sintéticos (VASSEUR et al., 1996; SELMI et al., 2002). Independentemente da técnica empregada para estabilização articular após a RLCCr, os resultados são clinicamente satisfatórios (MOORE \& READ, 1996), apesar de terem sido relatadas instabilidade articular decorrente do afrouxamento prematuro do substituto ligamentar e abrasão de implantes em contato com superfícies de túneis ósseos (VASSEUR et al., 1996; SELMI et al., 2002) ou em contato com a incisura troclear durante a extensão articular (HOWELL \& TAYLOR, 1993; FITCH et al., 1995a; FITCH et al., 1995b; MONTGOMERY et al., 1995; VASSEUR et al., 1996; LA PRADE et al., 1998; SELMI et al., 2002). Para minimizar estas últimas complicações, sugere-se a realização da incisuroplastia troclear, que consiste na remoção do aspecto medial do côndilo lateral do fêmur, em especial quando a inserção tibial do substituto ligamentar estiver localizada cranialmente à inserção do ligamento cruzado cranial (HOWELL \& TAYLOR, 1993; LA PRADE et al., 1998; MANN et al., 1999) ou quando há estenose intercondilar secundária à RLCCr (AIKEN et al., 1995; FITCH et al., 1995a; FITCH et al., 1995b; MONTGOMERY et al., 1995; LA PRADE et al., 1998; MANN et al., 1999).

Neste trabalho, objetivou-se avaliar, clínica e radiograficamente, a estabilização articular empregando retalho de fáscia lata, associado ou não à incisuroplastia troclear, em cães após a transecção do ligamento cruzado cranial.

\section{MATERIAL E MÉTODOS}

Foram utilizados nove cães hígidos, sem raça definida, machos ou fêmeas, adultos, com massa corporal entre 15 e 25kg $\pm 20,9$ (4,8kg), provenientes do Centro de Controle de Zoonoses. Os animais foram colocados em canis individuais com aproximadamente $1,5 m^{2}$ de área, onde receberam ração comerciala ${ }^{\mathrm{e}}$ água.

Os cães tiveram o joelho direito submetido à ITR e posterior estabilização articular (GI), enquanto que o joelho esquerdo foi submetido somente à substituição ligamentar (GC). Os animais foram agrupados em três subgrupos correspondentes ao período de eutanásia no 30ํㅜ 90ํe $180^{\circ}$ dia pósoperatório.

Para realização dos procedimentos cirúrgicos, os cães foram mantidos em jejum hídrico e alimentar nas 12 horas que antecederam o ato cirúrgico. A medicação pré-anestésica foi realizada com maleato de acepromazina ${ }^{\mathrm{b}}\left(0,1 \mathrm{mg} \mathrm{kg}^{-1}-\mathrm{IV}\right)$. A anestesia cirúrgica foi induzida com propofol ${ }^{\mathrm{c}}\left(6 \mathrm{mg} \mathrm{kg}^{-1}-\mathrm{IV}\right) 15$ minutos após. Os animais foram medicados com cetoprofeno ${ }^{\mathrm{d}}$ (2,2mg kg-1 - IV), e posicionados para realização de anestesia epidural, empregando-se sulfato de morfina ${ }^{\mathrm{e}}$ associado ao cloridrato de lidocaína ${ }^{\mathrm{f}}$ e de bupivacaína $^{\mathrm{g}}$, nas doses de $0,1,1,0$ e $0,5 \mathrm{mg} \mathrm{kg}^{-1}$, respectivamente. Os animais foram intubados e a anestesia mantida em circuito semifechado empregando-se sevoflurano ${ }^{\mathrm{h}}$ vaporizado em oxigênio a $100 \%$. Em seguida, foi administrada cefazolina sódica ${ }^{\mathrm{i}}\left(20 \mathrm{mg} \mathrm{kg} \mathrm{kg}^{-1}-\mathrm{IV}\right)$. Durante todo o período anestésico, os pacientes receberam solução de Ringer com lactato (10mL kg $\left.{ }^{-1} \mathrm{~h}^{-1}-\mathrm{IV}\right)$.

Abordou-se a articulação a partir de incisão parapatelar lateral. Após identificação, secção e remoção do LCCr, realizou-se no GI a incisuroplastia troclear, que consistiu no desgaste da porção medial do côndilo lateral do fêmur, com auxílio de fresa óssea acoplada a motor elétrico de baixa rotação. Removeuse a porção cranial, média e caudal da incisura troclear lateral, acompanhada da irrigação intermitente da articulação com solução salina estéril. Foi então confeccionado o retalho de fáscia lata, conforme técnica descrita por COETZEE \& LUBBE (1995). O retalho foi mantido unido à tuberosidade da tíbia, seguido de sua condução craniocaudal por sob o ligamento intermeniscal e, posteriormente, através da articulação com emergência caudal e proximal à fabela lateral. Para fixação do retalho, empregou-se fio de 
polipropileno n. $0^{j}$, com padrão de sutura interrompido simples. A cápsula articular foi suturada com fio de poliglactina n. $2-0^{\mathrm{k}}$, e o defeito na fáscia lata aproximado com pontos interrompidos simples com fio de polipropileno n. 2-01. A porção remanescente do retalho foi suturada sob tensão ao tendão patelar, empregando-se pontos interrompidos simples com polipropileno n. 0. A sutura do tecido subcutâneo foi realizada com padrão contínuo simples empregandose poliglactina n. 2-0, e o tecido cutâneo suturado com padrão interrompido simples utilizando-se náilon n. 3$0^{\mathrm{m}}$. No GC, o procedimento cirúrgico foi análogo, exceto pela ausência da incisuroplastia troclear. Para se minimizarem quaisquer efeitos temporais, as cirurgias nas articulações contralaterais foram realizadas em momentos cirúrgicos subseqüentes.

Foi aplicado adesivo de liberação gradativa de fentanil ${ }^{\mathrm{n}}$ à região lombar, imediatamente ao término da cirurgia. Diariamente, efetuou-se limpeza da ferida cirúrgica com solução fisiológica. A administração de cetoprofeno foi realizada na mesma dose que a utilizada previamente à cirurgia durante quatro dias subseqüentes ao ato cirúrgico. A retirada dos pontos foi executada no décimo dia pós-operatório.

As variáveis clínicas avaliadas referiam-se ao grau de claudicação; à presença do movimento de gaveta em extensão articular passiva máxima e flexão de $90^{\circ}$; ao deslocamento cranial da tíbia; ao perímetro da coxa; aos graus máximos de extensão e flexão passiva e amplitude de movimento articular; e ao grau de rotação interna da tíbia. Radiograficamente avaliouse o grau de doença articular degenerativa (DAD). Todas as variáveis foram avaliadas previamente à cirurgia (T -1), no pós-operatório imediato (T1) e aos 30 (T30), 90 (T90) e 180 (T180) dias de pós-operatório, dependendo do subgrupo em estudo.

O grau de claudicação foi determinado por meio de escores, sendo considerado o valor 0 na ausência de claudicação, 1 para claudicação após exercícios, 2 para claudicação ao caminhar e correr, 3 para claudicação ao caminhar, sem apoio ao correr, e 4 para impotência funcional (AIKEN et al., 1992). O movimento de gaveta das articulações do joelho foi quantificado como 0 para articulações estáveis, 1 para articulações com movimento de gaveta presente, porém limitado, e 2 para joelhos com movimento de gaveta evidente. O deslocamento cranial da tíbia foi observado mediante o teste de compressão tibial e quantificado de forma análoga ao movimento de gaveta. O perímetro da coxa foi mensurado com auxílio de fita maleável de escala métrica, graduada em centímetros, na altura da prega inguinal. Os graus máximos de extensão e flexão passivas das articulações do joelho foram mensurados por meio de goniômetro (JAEGGER et al., 2002), assim como o grau máximo de rotação interna da tíbia.

O exame radiográfico dos joelhos foi realizado nas posições mediolateral e craniocaudal, nos momentos T-1, T1, T30, T90 e T180. As radiografias foram analisadas, de forma que cada alteração radiográfica foi graduada com escore de acordo com a sua gravidade. Nessa escala, 0 representava ausência de alterações, e 1, 2 e 3 representavam alterações leves, moderadas e graves, respectivamente. Baseada no método de VASSEUR \& BERRY (1992), a soma dos escores atribuídos para cada alteração presente indicou a intensidade de DAD para cada articulação.

Para avaliação das variáveis nos momentos pós-operatórios, excetuando-se o grau de claudicação, os animais foram tranqüilizados com maleato de acepromazina $1 \%\left(0,1 \mathrm{mg} \mathrm{kg}^{-1}-\mathrm{IV}\right)$ e, após 15 minutos, anestesiados com tiopental sódico ${ }^{\circ}\left(12,5 \mathrm{mg} \mathrm{kg}^{-1}-\mathrm{IV}\right)$.

Para comparação dos dados, utilizou-se delineamento inteiramente casualisado, considerandose os efeitos das técnicas cirúrgicas e do tempo de avaliação, para cada variável estudada. Foi utilizado o teste de Friedman para a comparação dos dados nãoparamétricos e o teste de Tukey para a dos dados paramétricos. Todos os testes foram realizados valendo-se do nível de significância de $1 \%$ de probabilidade.

\section{RESULTADOS E DISCUSSÃO}

A importância do estudo das alterações decorrentes de técnicas intra-articulares, após ruptura ou transecção experimental do ligamento cruzado cranial (LCCr), deve-se à alta freqüiência desta condição na clínica de pequenos animais (AIKEN et al., 1992). A incisuroplastia troclear (ITR) é comumente associada às técnicas cirúrgicas em seres humanos, especialmente quando a estenose da fossa intercondilar é observada no momento trans-operatório (MONTGOMERY et al., 1995; HOWELL \& TAYLOR, 1993; MANN et al., 1999). De acordo com AIKEN et al. (1995), VASSEUR et al. (1996), MANN et al. (1999) e SELMI et al. (2002), a estenose intercondilar pode sujeitar o substituto ligamentar a abrasão e ruputra precoce, e limitar a extensão articular.

Neste estudo, imediatamente antes da cirurgia, não foram observadas diferenças significativas entre os grupos para as variáveis analisadas. Durante o ato operatório, observou-se a presença e integridade do LCCr em todas as articulações. Nenhum animal apresentava alterações da marcha no momento préoperatório; entretanto, o grau de claudicação variou de forma significativa no primeiro momento de avaliação

Ciência Rural, v.37, n.5, set-out, 2007. 
pós-operatória (T1) em ambos os grupos (GI, - P<0,01, GC - $\mathrm{P}<0,01)$ sem, no entanto, haver diferenças entre os grupos. Nos momentos subseqüentes da avaliação, foi notado discreto grau de claudicação em ambos os grupos, todavia, aos seis meses de avaliação, considerou-se a marcha como sendo normal.

A avaliação do grau de claudicação pósoperatória tem sido objeto de vários estudos empregando diferentes técnicas para estabilização articular, após a RLCCr(SHIRES \& HULSE, 1984;AIKEN et al., 1992; COETZEE \& LUBBE, 1995; FITCH et al., 1995b; VASSEUR et al., 1996; GEELS et al., 2000; SELMI et al., 2002). Neste estudo, foi observada melhora progressiva do grau de claudicação, a partir dos 30 dias de pós-operatório, em ambos os joelhos, de modo que os escores relacionados não diferiam estatisticamente dos valores pré-operatórios, tampouco entre os grupos. Porém, do ponto de vista clínico, pôde-se observar discreta claudicação nos animais em estudo durante os intervalos de avaliação, exceto aos 180 dias pós-operatórios. Esta discrepância também foi observada por MELO (2001) e SELMI et al. (2002). Possivelmente, a avaliação subjetiva da claudicação é susceptível a fatores como condicionamento do animal, ambiente de avaliação e dor. Dessa forma, a melhora nos escores de claudicação pode ser resultante da adaptação do animal às condições experimentais impostas ou pelo deslocamento do centro de gravidade, ou ainda expressar a estabilidade articular obtida após substituição ligamentar, conforme sugeriram SHIRES \& HULSE (1984) e COTZEE \& LUBBE (1995).

A ausência de diferença estatística observada nos escores de claudicação é corroborada pelos achados relativos à estabilidade articular, conforme técnica sugerida por COETZEE \& LUBBE (1995). Em ambos os joelhos, o movimento de gaveta, durante a extensão articular, não diferiu significativamente entre os grupos ao longo do tempo, enquanto que, durante a flexão, as articulações apresentavam-se instáveis entre T1 e T90; em T180, as articulações apresentavam-se com discreta instabilidade, entretanto sem diferenças estatísticas com relação aos valores pré-operatórios ou entre os grupos. Este achado está relacionado à aproximação da origem e da inserção do retalho de fáscia lata durante a flexão articular, resultando em afrouxamento intraarticular desta estrutura. Tal observação se assemelha às condições relatadas por ARNOCSKY (1996), que descreve o LCCr como sendo um aglomerado de feixes com comportamento independente durante os diferentes graus da amplitude articular, ficando ora tensos, ora frouxos. Outrossim, a ausência de significância estatística observada em T180 pode ser resultado da fibrose periarticular, decorrente da instabilidade crônica.

Segundo SHIRES \& HULSE (1984) e ARNOCSKY (1996), o posicionamento “over-the-top” possibilita a manutenção da isometricidade dos substitutos ligamentares, contribuindo para a estabilização articular durante a amplitude articular. A falta de estabilidade clínica observada durante a flexão articular nos cães deste estudo pode estar relacionada ao posicionamento supracitado inadequado do substituto ligamentar. Desta forma, o retalho de fáscia lata estaria direcionado através de diferentes áreas sobre o aspecto caudodorsal do côndilo lateral do fêmur, com posicionamento variado entre os animais.

De acordo com INNES \& BARR (1998), a sedação ou anestesia é fundamental para avaliação clínica, já que diferenças nos graus de sedação podem resultar em variações inaceitáveis ao longo do tempo, para um mesmo indivíduo. MELO (2001) atribuiu a ausência de diferenças estatísticas na amplitude de movimento e circunferência da coxa à falta de sedação adequada e conseqüente não-colaboração do paciente durante a avaliação clínica. Contrariamente, alguns autores relataram falta de diferenças entre variáveis clínicas com os animais sob efeito de sedativos ou anestésicos (SHIRES \& HULSE, 1984; LA PRADE et al., 1998; JAEGGER et al., 2002). Esta variação entre estudos sugere que o relaxamento muscular não influencia nos resultados do exame clínico, e que estes estão relacionados diretamente ao sucesso da técnica cirúrgica empregada. Corroboram essa afirmação os achados deste estudo, no qual as variáveis clínicas foram determinadas após anestesia dos animais, sendo observadas diferenças estatísticas entre o diâmetro muscular da coxa em T30 e T90 ( $<<0,01)$, nos animais submetidos à ITR ou não. Para LA PRADE et al. (1998), a ITR não produziu alterações no diâmetro muscular ou na amplitude de movimento do joelho; entretanto, os autores realizaram a ITR em articulações estáveis, o que contribuiu para manutenção da estabilidade articular e uso adequado do membro.

De acordo com INNES \& BARR (1998), a condição da musculatura da coxa é o melhor indicativo do sucesso a longo prazo de técnicas que visam a restabelecer a estabilidade articular. Neste estudo, pôdese observar que, após declínio dessa massa muscular, de forma indistinta entre os grupos em T30 e T90, houve início de recuperação em T180, condição concordante com a observação clínica de normalidade da deambulação.

A amplitude de movimento articular é comumente utilizada como forma de avaliação póscirúrgica (SHIRES \& HULSE, 1984; LA PRADE et al., 
1998; MELO, 2001), porém, não deve ser analisada isoladamente, uma vez que aumento no grau de extensão ou flexão articular acompanhado por redução do movimento antagônico pode produzir tal parâmetro de formas similares. Assim, quando considerados os graus máximos de extensão e flexão articulares, e a amplitude articular passiva, avalia-se a adequação da articulação ao procedimento cirúrgico. Ainda, visto a interação das diversas estruturas articulares na manutenção da estabilidade, faz-se necessário determinar o grau de rotação interna presente nos joelhos, uma vez que o LCCr possui a função de limitar a excessiva rotação interna da tíbia durante a flexão. A manutenção dos graus de rotação interna da tíbia, da extensão e flexão articulares, assim como da amplitude de movimento, nos diferentes grupos, durante todo o período de avaliação, sugere que a técnica descrita por COETZEE \& LUBBE (1995) propicia adequada estabilidade pós-operatória, e que a ITR não altera os movimentos articulares. Entretanto, deve-se considerar a confecção do retalho extra-articular de fáscia lata, que pode ter funcionado como uma faixa restritiva à rotação interna da tíbia, conforme descreveram AIKEN et al. (1992) e COETZEE \& LUBBE (1995).

A despeito das citações de FITCH et al. (1995a) e MONTGOMERY et al. (1995), que descreveram que, durante a extensão do joelho, o LCCr entra em contato com o teto e a porção lateral da abertura cranial da fossa intercondilar, limitando a hiperextensão, não foram observadas diferenças no grau máximo de extensão articular entre os grupos. Entretanto, numericamente, os joelhos submetidos à ITR apresentavam maiores valores de extensão articular.

De acordo com HOWELL \& TAYLOR (1993)

e MANN et al. (1999), o posicionamento cranial de substitutos ligamentares, em relação à tíbia, propicia cargas de tensão excessivas durante a extensão articular devido ao contato do "neoligamento" com o teto e a parede lateral da fossa intercondilar. Após RLCCr, a estenose da fossa intercondilar pode sujeitar o substituto ligamentar a abrasão ou limitação na extensão articular (VASSEUR et al.,1996; MANN et al., 1999; SELMI et al., 2002). Vários fatores devem ser considerados quando do planejamento de técnicas intra-articulares seguidas de ITR, entre eles a localização tibial do substituto ligamentar, o grau de estenose intercondilar e a largura do "neoligamento" (MANN et al., 1999). A despeito do posicionamento cranial do retalho de fáscia lata, não foi observada redução da extensão articular em nenhum dos animais deste estudo, independentemente da realização da ITR, tampouco instabilidade articular que pudesse sugerir a ruptura precoce do "neoligamento".
Apesar de não terem sido observadas diferenças significativas entre os grupos, nos subseqüentes momentos de avaliação, pôde-se notar comportamento semelhante para as variáveis amplitude de movimento, grau de extensão e flexão máxima articular e rotação interna da tíbia, que apresentaram valores numéricos inferiores aos 30 dias, concordando com as observações de maior grau de claudicação e menor perímetro da coxa observado naquele momento da avaliação, em ambos os grupos.

As alterações radiográficas mais freqüentemente observadas em ambas as articulações foram efusão e distensão da cápsula articular, presentes desde o momento pós-operatório imediato até o término deste estudo. $\mathrm{O}$ espessamento dos tecidos moles periarticulares foi observado a partir dos 30 dias após o procedimento, sendo mais evidente no aspecto medial das articulações. A presença de osteófito na base ou ápice da patela e nas cristas trocleares foi observada a partir dos 30 dias e apresentou variação indistinta entre os grupos nos diferentes momentos de observação subseqüentes. Apesar do aumento dos escores atribuídos à progressão da DAD até os 90 dias de avaliação pós-operatória e posterior manutenção, possivelmente decorrente da estabilidade proporcionada pela fibrose periarticular, a somatória das alterações radiográficas observadas não diferiu estatisticamente entre os grupos durante os momentos de avaliação.

Para ELKINS et al. (1991), a observação radiográfica de osteófitos pode ser realizada já aos 21 dias de pós-operatório. Neste estudo, o aparecimento de osteófitos na base da patela e nas cristas trocleares, logo aos 30 dias de avaliação, confirma observações anteriores empregando a técnica "under-and-over" descrita inicialmente por SHIRES \& HULSE (1984), e posteriormente empregada por ELKINS et al. (1991), VASSEUR\&BERRY(1992), COETZEE \& LUBBE(1995) e GEELS et al. (2000). Comparações entre técnicas intra ou extra-articulares, tangentes à progressão da DAD, não demonstram superioridade de uma em detrimento da outra (ELKINS et al., 1991), assim como estudos voltados à comparação de técnicas intra ou extraarticulares (COETZEE \& LUBBE, 1995). Assim, a escolha do procedimento de estabilização articular depende da familiaridade do cirurgião com a técnica empregada (MOORE \& READ, 1996, INNES \& BARR, 1998; GEELS et al. 2000).

Relativamente à ITR, FITCH et al. (1995b) e LA PRADE et al. (1998) observaram ausência de alterações degenerativas seis meses após sua realização em joelhos caninos estáveis. Em contra-partida, em joelhos instáveis, após transecção do LCCr, FITCH et 
al. (1995b) relataram a extensa formação de osteófitos, sugerindo degeneração articular. Neste estudo, independentemente do aumento dos escores relativos à $\mathrm{DAD}$, até os 90 dias de avaliação, não foram observadas diferenças significativas entre os grupos ao longo do tempo, mormente pelo fato da estabilização articular ter sido realizada imediatamente após transecção do LCCr, ou pela falta de processos patológicos pré-existentes, ou até mesmo por possível confinamento dos cães.

\section{CONCLUSÕES}

Baseados nas alterações encontradas, para as variáveis clínicas e radiográficas, conclui-se que a associação da incisuroplastia troclear à reconstrução intra-articular com retalho de fáscia lata não resulta em agravamento da função articular, tampouco produz alterações radiográficas significativas, semelhantemente à estabilização intra-articular com fáscia lata, isoladamente. Entretanto, independentemente da técnica cirúrgica empregada, o retorno à normalidade da deambulação e do perímetro da coxa só pôde ser observado após 180 dias da intervenção.

\section{FONTESDEAQUISIÇÃO}

${ }^{a}$ Golden Formula, Grandfood do Brasil. SP.

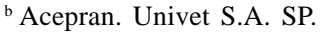

${ }^{\text {c }}$ Propoabbott. Abbott do Brasil. SP.

${ }^{\mathrm{d}}$ Ketofen. Merial. SP.

e Dimorf. Cristália. SP.

${ }^{\mathrm{f}}$ Xilestesin. Cristália. SP.

g Bupivacaína. Cristália. SP.

h Sevorane. Abbott do Brasil. SP.

${ }^{\mathrm{i}}$ Kefazol. Elli-Lilly. SP.

j Prolene. Ethicon. SP.

k Vicryl. Ethicon. SP.

${ }^{1}$ Prolene. Ethicon. SP.

${ }^{\mathrm{m}}$ Mononylon. Ethicon. SP.

${ }^{n}$ Durogesic. Jansen \& Cillag. Canadá.

${ }^{\circ}$ Thionembutal. Abbott do Brasil. SP

\section{COMITÊ DE ÉTICA E BIOSSEGURANÇA}

O trabalho foi submetido e aprovado pela Comissão de Ética da Universidade de Brasília, e realizado de acordo com as normas do COBEA.

\section{REFERÊNCIAS}

AIKEN, S.W. et al. Extra-articular fascial strip repair of the cranial cruciate deficient stifle: technique and results in seven dogs. Veterinary and Comparative Orthopedics and Traumatology, v.5, p.145-150, 1992.

AIKEN, S.W. et al. Intercondylar notch width in dogs with and without cranial cruciate ligament injuries. Veterinary and
Comparative Orthopedics and Traumatology, v.8, p.128132, 1995

ARNOCSKY, S.P. Patomecânica das lesões do ligamento cruzado e meniscos. In: BOJRAB, M.J. Mecanismos da moléstia de pequenos animais. 2.ed. São Paulo: Manole, 1996. Cap.110, p.889-902.

COETZEE, G.L.; LUBBE, A.M. A prospective study comparing two fascial reconstruction techniques to stabilize the cranial cruciate deficient stifle in the dog. Veterinary and Comparative Orthopedics and Traumatology, v.8, p.8290, 1995.

ELKINS, A.D. et al. A retrospective study evaluating the degree of degenerative joint disease in the stifle joint of dogs following surgical repair of anterior cruciate ligament rupture. Journal of the American Animal Hospital Association, v.27, p.533540, 1991

FITCH, R.B. et al. The intercondylar fossa of the normal canine stifle: an anatomic and radiographic study. Veterinary Surgery, v.24, p.148-155, 1995a.

FITCH, R.B. et al. The effect of intercondylar notchplasty on the normal canine stifle. Veterinaty Surgery, v.24, p.156164, $1995 b$.

GEELS, J.J. et al. Evaluation of an intracapsular technique for the treatment of cranial cruciate ligament rupture. Veterinary and Comparative Orthopedics and Traumatology, v.13, p.197-203, 2000.

HOWELL, S.M.; TAYLOR, M.A. Failure of reconstruction of the anterior cruciate ligament due to impingement by the intercondylar roof. Journal of Bone and Joint Surgery, v.75-A, n.7, p.1044-1055, 1993.

INNES, J.F.; BARR, A.R.S. Clinical natural history of the postsurgical cruciate deficient canine stifle joint: 1 year. Journal of Small Animal Practice, v.39, p.325-332, 1998.

JAEGGER, G. et al. Reliability of goniometry in Labrador Retrievers. American Journal of Veterinary Research, v.63, n.7, p.979-986, 2002.

LA PRADE, R.F. et al. The effects of aggressive notchplasty on the normal knee in dogs. American Journal of Sports Medicine, v.26, n.2, p.193-200, 1998.

MANN, T.A. et al. The natural history of the intercondylar notch after notchplasty. American Journal of Sports Medicine, v.27, n.2, p.181-188, 1999.

MELO, E.G. Sulfato de condroitina e hialuronato de sódio no tratamento da doença articular degenerativa em cães. Estudo experimental. 2001. 94f. Tese (Doutorado em Patologia e Ciências Clínicas) - Escola de Veterinária, Universidade Federal de Minas Gerais, Belo Horizonte.

MONTGOMERY, R.D. et al. Radiographic imaging of the canine intercondylar fossa. Veterinary Radiology and Ultrasound, v.36, n.4, p.276-282, 1995.

MOORE, K.W.; READ, R.A. Rupture of the cranial cruciate ligament in dogs - part II - diagnosis and management. 
Compendium of Continuing Education for the Practicing Veterinarian, v.18, n.4, p.381-391, 1996.

READ, R.A.; ROBINS, G.M. Deformity of the proximal tibia in dogs. Veterinary Record, Londres, v.111, p.295-298, 1982.

SELMI, A.L. et al. Clinical radiographic evaluation of a polyester prosthesis in dogs with cranial cruciate ligament rupture. Ciência Rural. v.32, n.5, p.793-798, 2002.

SHIRES, P.K.; HULSE, D.A. The under-and-over fascial replacement technique for anterior cruciate ligament rupture in dogs: a retrospective study. Journal of the American Animal Hospital Association, v.20, p.69-77, 1984.
SLOCUM, B.; DEVINE, T. Cranial tibial thrust: a primary force in the canine stifle. Journal of the American Veterinary Medical Association, v.183, p.456-466, 1983.

VASSEUR, P.B.; BERRY, C.R. Progression of stifle osteoarthrosis following reconstruction of the cranial cruciate ligament in 21 dogs. Journal of the American Animal Hospital Association, v.28, p.129-136, 1992.

VASSEUR, P.B. et al. Evaluation of the Leeds-Keio synthetic replacement for the cranial cruciate ligament in dogs: an experimental study. Veterinary and Comparative Orthopedics and Traumatology, v.9, p.66-74, 1996. 